\title{
High-Resolution Time-frequency Distributions for Fall Detection
}

\author{
Moeness G. Amin ${ }^{\mathrm{a}}$, Yimin D. Zhang ${ }^{\mathrm{a}}$, Boualem Boashash ${ }^{\mathrm{b}}$ \\ ${ }^{a}$ Center for Advanced Communications, Villanova University, Villanova, PA 19085, USA \\ ${ }^{\mathrm{b}}$ Department of Electrical Engineering, College of Engineering, Qatar University, Doha, Qatar
}

\begin{abstract}
In this paper, we examine the role of high-resolution time-frequency distributions (TFDs) of radar micro-Doppler signatures for fall detection. The work supports the recent and rising interest in using emerging radar technology for elderly care and assisted living. Spectrograms have been the de facto joint-variable signal representation, depicting the signal power in both time and frequency. Although there have been major advances in designing quadratic TFDs which are superior to spectrograms in terms of detailing the local signal behavior, the contributions of these distributions in the area of human motion classifications and their offerings in enhanced feature extractions have not yet been properly evaluated. The main purpose of this paper is to show the effect of using high-resolution TFD kernels, in lieu of spectrogram, on fall detection. We focus on the extended modified B-distribution (EMBD) and exploit the level of details it provides as compared with the coarse and smoothed time-frequency signatures offered by spectrograms.
\end{abstract}

Keywords: Fall detection, assisted living, time-frequency distribution, classification

\section{INTRODUCTION}

The old-age dependency ratio, which is defined as the ratio of the population aged 65 years or over to the population aged 20-64, has been rising in many countries all over the world. According to United Nations estimates for about 30 countries, this ratio is anticipated to reach $30 \%$ in 2020 [1]. A number of techniques have been proposed to sense vital signs and motions for ambient assisted living of elderly [2-4]. Exiting sensing techniques include those monitoring physiological signs (e.g., electrocardiography (ECG)) and accelerometers, camera and thermography, passive infrared sensors, and radio frequency identification (RFID) devices. It is noted that ECG, accelerometers, and RFID require that devices be attached to human body, whereas camera systems are sensitive to lighting conditions, may be obscured by walls and fabrics, and, more importantly, raise privacy concerns.

Radar is an excellent sensing modality for assisted living and has gain rapidly increasing interests [5-9]. Radars offer non-intrusive, clutter suppressed and noise tolerant sensing systems [10]. As such, they enable monitoring of the elderlies' motion profiles in their private homes. Radar systems avoid direct contact (unlike ECG and accelerometers). Compared to optical and infrared-based systems, radar systems can operate in all types of environments, can penetrate walls and fabrics, preserve privacy, and are insensitive to lighting conditions.

A radar system transmits an electromagnetic wave over a certain range of frequencies and analyzes the return signals. It estimates the velocity of a moving object by measuring the frequency shift of the wave radiated or scattered by the object, known as the Doppler effects [11]. For an articulated object such as a walking person, the motion of various components of the body including arms and legs induces frequency modulations on the returned radar signal and generates sidebands about the Doppler frequency, referred to as micro-Doppler signatures [12]. Radar Micro-Doppler is the phenomenon of the observed micro-motions on top of the bulk main Doppler component of a target's motion. It can be observed by continuous-wave (CW), stepped-frequency continuous-wave (SFCW), or pulse-Doppler radar systems [13-16]. Whereas the motion of the torso creates a Doppler frequency, the motions of the limbs cause micro-Doppler which comprises the changes around the Doppler frequency.

Motion classifications based on target micro-Doppler signatures are typically based on time-frequency analyses of the radar returns. Among quadratic TFDs, spectrograms have been the de facto joint-variable signal representation, depicting the signal power in both time and frequency $[5,6,8,14]$. The wavelet transform, as a linear signal time-frequency representation, is also found useful for this purpose because of its use of spectrum-dependent window functions [7, 9].

*Contact information: yimin.zhang@villanova.edu. 
Several papers on motion classifications using radar proceed to extract pertinent features to the motion profiles of interest in the time-frequency domain and then apply these features to a classifier. Although there have been major advances in designing quadratic time-frequency distributions (TFDs) which are superior to spectrograms in terms of detailing the local signal behavior, the contributions of these distributions in the area of human motion classifications and their offerings in enhanced feature extractions have not yet been properly evaluated.

In this paper, we examine the role of high resolution TFDs for fall detection. The work supports the recent and rising broad interest in using emerging radar technology for elderly care and assisted living. The paper contributions are twofold: a) We show the effect of using high resolution TFDs, in lieu of spectrograms on classification rates of the fall. We use features which have been commonly identified and used in association of falls and spectrograms; b) We devise new features in congruence with high resolution TFDs which exploit the level of details provided by these distributions in comparison with the coarse and smoothed time-frequency signatures offered by spectrograms.

\section{SIGNAL MODEL}

A monostatic CW radar transmits a sinusoidal signal, expressed as $s(t)=\exp \left(j 2 \pi f_{c} t\right)$, where $f_{c}$ is the carrier frequency. Consider a point target which is located at a distance of $R_{0}$ from the radar at time $t=0$, and moves with a velocity of $v(t)$ in a direction forming an angle of $\theta$ with the radar line-of-sight. As such, the distance between the radar and the target at time instant $t$ is

$$
R(t)=R_{0}+\int_{0}^{t} v(u) \cos (\theta) d u .
$$

The received radar signal can be expressed as

$$
x_{a}(t)=\rho \exp \left[j 2 \pi f_{c}\left(t-\frac{2 R(t)}{c}\right)\right],
$$

where $\rho$ is the target reflection coefficient and $c$ is the velocity of the EM wave propagation. The Doppler frequency corresponding to $x_{a}(t)$ is

$$
f_{D}(t)=2 v(t) \cos (\theta) / \lambda_{c}
$$

where $\lambda_{c}=c / f_{c}$ is the wavelength.

For a spatially extended target, such as a human body, the radar return is the integration over the target region $\Omega$, given by

$$
x(t)=\int_{\Omega} x_{a}(t) d a .
$$

In this case, the Doppler signature is the superposition of all component Doppler frequencies. Torso or gait motions generally generate time varying Doppler frequencies, and their exact signatures depend on the target shape and motion patterns. Note that, as the frequency varies with time, this type of signals is usually characterized as nonstationary FM signals.

\section{HIGH-RESOLUTION TIME-FREQUENCY ANALYSIS}

\subsection{Time-Frequency Distribution}

Time-frequency analysis is a natural tool that reveals the time-varying Doppler and micro-Doppler frequency signatures with enhanced signal energy concentration. A number of methods are available to perform time-frequency analysis of the Doppler and micro-Doppler signatures. These methods can be generally divided into the following two classes: linear time-frequency analysis and quadratic time-frequency analysis. The short-time Fourier transform (STFT) is a commonly used technique to perform linear time-frequency analysis [17]. Its squared magnitude, referred to as the spectrogram, is related to a large class of quadratic time-frequency representations [18].

The STFT of the data $x(t)$ with a window $h(t)$ is expressed as 


$$
S(t, f)=\sum_{m=-\infty}^{\infty} h(m) x(t-m) \exp (-j 2 \pi f m)
$$

where $f$ is the frequency index. The spectrogram is obtained by computing the squared magnitude of STFT, expressed as

$$
D(t, f)=|S(t, f)|^{2}=\left|\sum_{m=-\infty}^{\infty} h(m) x(t-m) \exp (-j 2 \pi f m)\right|^{2} .
$$

On the other hand, the quadratic class of TFD of a signal $x(t)$ is defined as the two-dimensional Fourier transform of its kernelled ambiguity function, expressed as

$$
D(t, f)=\sum_{\theta=-\infty}^{\infty} \sum_{\tau=-\infty}^{\infty} \phi(\theta, \tau) A(\theta, \tau) \exp (j 4 \pi f \tau-j 2 \pi \theta t),
$$

where

$$
A(\theta, \tau)=\sum_{u=-\infty}^{\infty} x(u+\tau) x^{*}(u-\tau) \exp (-j 2 \pi \theta u)
$$

is the ambiguity function, and $\phi(\theta, \tau)$ is the time-frequency kernel. Here, $\theta$ and $\tau$, respectively, denote the frequency shift (also referred to as Doppler frequency) and the time lag. The properties of a quadratic TFD are heavily affected by the applied kernel.

The Wigner-Ville distribution (WVD) is often regarded as the basic or prototype quadratic TFD, since the other class of quadratic TFDs can be described as filtered version of the WVD, whereas the effective kernel function of the WVD can be considered as unity across the entire ambiguity function. WVD is known to provide the best time-frequency resolution for single-component linear frequency modulated (LFM) signals, but it yields undesirable cross-terms when the frequency law is nonlinear or when a multi-component signal is considered.

Various reduced-interference kernels (RIDs) have been developed to reduce the cross-term interference. RID kernel $\phi(\theta, \tau)$ acts as a filter and places different weightings on the ambiguity function. Majority of signals have auto-terms located near the origin in the ambiguity domain, while the signal cross-terms are distant from the time-lag and frequency-shift axes. As such, RID kernels exhibit low-pass filter characteristics to suppress cross-terms and preserve auto-terms.

\subsection{Extended Modified B-Distribution}

High-resolution kernels have been a subject of interest in bilinear time-frequency analysis [18-20]. In this paper, we use the extended modified B-distribution (EMBD) to generate the TFDs of the radar returns. It has been shown that EMBD is the simplest of several TFDs that achieve better auto-term resolution and yields improved performance for target classification [18]. The EMBD applies its kernel filter along both lag and Doppler axes, resulting in the expression [20]:

$$
\phi(\theta, \tau)=\frac{|\Gamma(\beta+j \pi \theta)|^{2}}{\Gamma^{2}(\beta)} \frac{|\Gamma(\alpha+j \pi \tau)|^{2}}{\Gamma^{2}(\alpha)},
$$

where $-0.5 \leq \theta \leq 0.5,-0.5 \leq \tau \leq 0.5,0 \leq \alpha \leq 1$, and $0 \leq \beta \leq 1$. The lengths of the Doppler and lag windows are controlled by separate parameters $\alpha$ and $\beta$, respectively. The extra degrees of freedom in the formulation of the EMBD allow to independently adjust the lengths of the windows along both lag and Doppler axes. This renders it a more useful tool for the analysis of the gait signals considered in this paper. Since we deal with deterministic signals, the timefrequency kernel is not tasked with producing low variance spectral estimate which is the case in nonstationary random processes $[21,22]$.

\section{EXPERIMENTAL RESULTS}

\subsection{Experimental Setup}

A CW radar was set up in the Radar Imaging Lab at Villanova University. The experiment scene is shown in Fig. 1. 
Agilent E5071B network analyzer was used for signal generation and measurement of radar returns. A carrier frequency of $8 \mathrm{GHz}$ was employed and the transmit power was set to $3 \mathrm{dBm}$. The network analyzer was externally triggered at a 1 $\mathrm{kHz}$ sampling rate. The record time of each experiment is 20 seconds, generating a total of 20,000 data samples. A vertically polarized horn antenna (BAE Systems, Model H-1479) with an operational frequency range of 1-12.4 GHz and 3-dB beamwidth of 45 degrees was used as a transceiver for the $\mathrm{CW}$ monostatic radar. The feed point of the antenna was positioned $1.15 \mathrm{~m}$ above the floor.

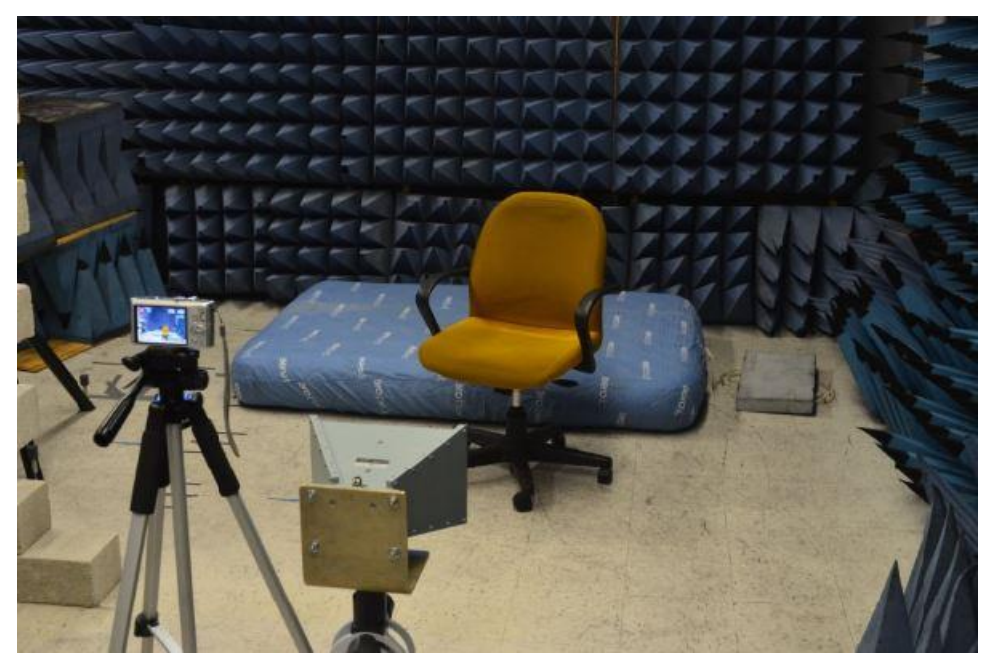

Figure 1. Experiment scene.

The radar data were collected from human subjects with direct line of sight to the targets. The experimental studies were approved by Villanova University's Institutional Review Board and with consent from all participants.

\subsection{Feature Extraction}

The feature extraction and motion classification were described in [8]. In this paper, we demonstrate that the use of $\mathrm{EMBD}$, in lieu of the spectrogram, enhances the feature extraction.

Based on the time-frequency analysis results obtained from either the spectrogram or the EMBD, the power burst curve $(\mathrm{PBC})$ is generated to identify important events for which a classification process should be initiated to detect whether such an event is a fall. The PBC represents the summation of signal power within a specific frequency band between frequencies $f_{1}$ and $f_{2}$ at the $k$ th time instant, i.e.,

$$
P B C[t]=\sum_{f \in\left[f_{1}, f_{2}\right]}|D(t, f)|^{2}+\sum_{f \in\left[-f_{2},-f_{1}\right]}|D(t, f)|^{2} .
$$

We choose $f_{1}=70 \mathrm{~Hz}$ and $f_{2}=100 \mathrm{~Hz}$ to detect high-power events as catastrophic events, such as falls, typically have high Doppler power content within this frequency band [8].

Once an event is detected by thresholding the PBC, we construct a four-second window of the spectrogram, centered around each of these points that correspond to the PBC peak, to determine whether a fall has happened. The proposed technique consists of segmentation processing to obtain a clean binary time-frequency signature for feature extraction in each window.

\subsection{Results}

Fig. 2 shows the spectrogram and EMBD of a fall towards the radar, generating positive Doppler frequencies at around $t$ $=8$ second. For the EMBD, parameters that control the Doppler and lag windows are chosen to be $\alpha=0.04$ and $\beta=0.5$ 
in all the computations. The threshold is respectively adjusted to filter out most of the noise, and the results are shown with the same $40 \mathrm{~dB}$ dynamic range. Note that the exact levels differ for the two distributions due to different scaling in their computations. For this example, a PBC peak is detected around $t=7.5$ second, and the corresponding four-second plots are shown in Fig. 3. When compared to the smoothed spectrogram, it is clear that the EMBD achieves a better contrast and connectivity, and reveals more details about the Doppler frequency signature.

We artificially add complex Gaussian noise to the observed data such that the noise floor is raised approximately by 5 $\mathrm{dB}$. The corresponding four-second TFD plots are shown in Fig. 4. The value range is raised by $5 \mathrm{~dB}$ in both plots as compared to Fig. 3. Fig. 5 shows the segmented binary image, without performing morphological processing, corresponding to the spectrogram and the EMBD, where the threshold level is $-54 \mathrm{~dB}$ for the spectrogram and $-29.5 \mathrm{~dB}$ for the EMBD. The segmented results allow the extraction of the useful features, e.g., extreme frequencies, extreme frequency ratio between positive and negative frequency components, and the length of event, for fall detection and motion classification [8]. The enhanced connectivity and the higher level of details provided by the EMBD are poised to provide more reliable feature extraction which will be verified once a high number of training and testing data is collected.

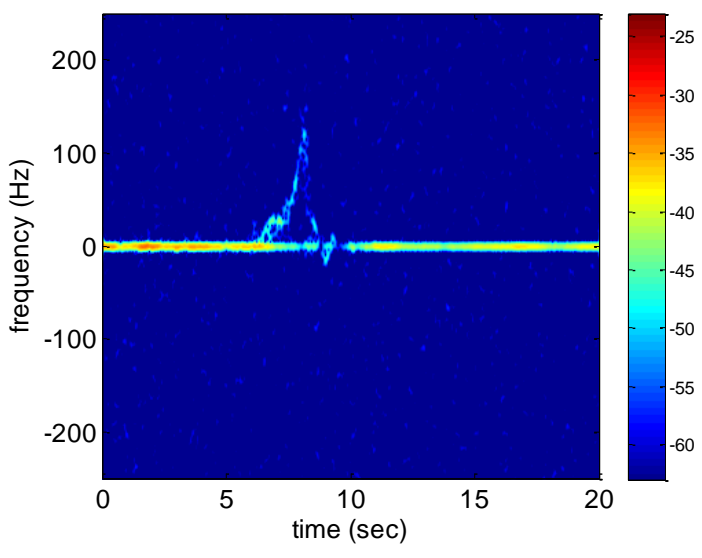

(a)

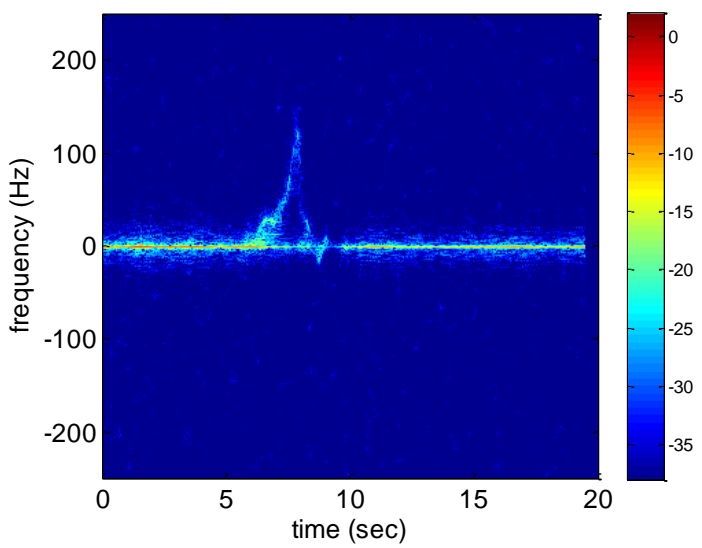

(b)

Figure 2. TFDs of falls. (a) Spectrogram; (b) EMBD.

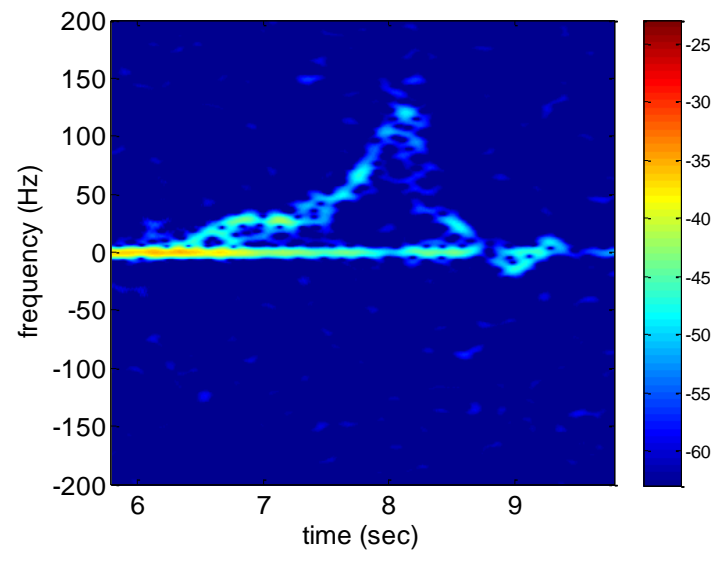

(a)

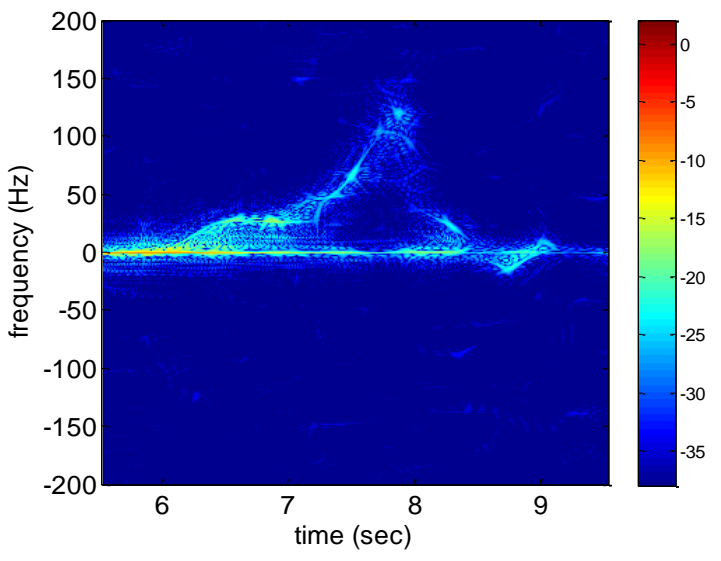

(b)

Figure 3. TFDs of falls for the four-second interval around the fall. (a) Spectrogram; (b) EMBD. 


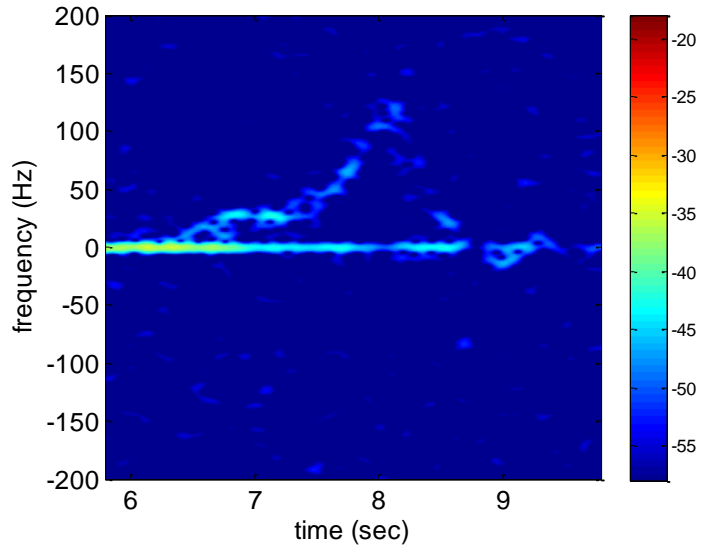

(a)

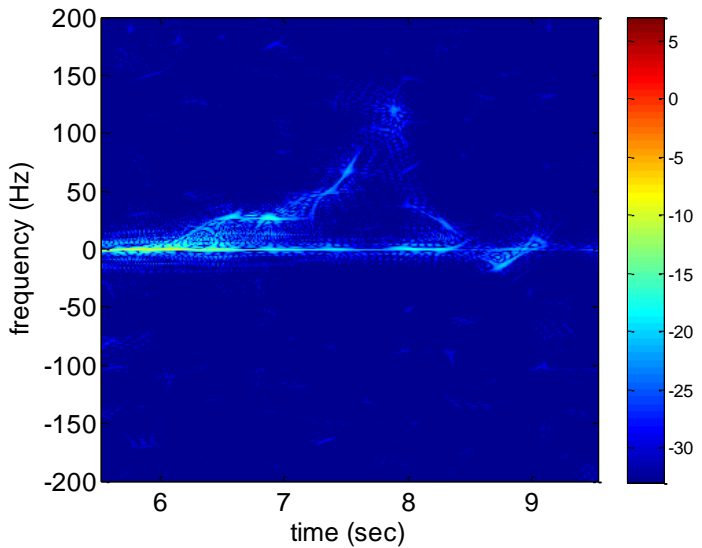

(b)

Figure 4. TFDs of falls with added artificial noise. (a) Spectrogram; (b) EMBD.

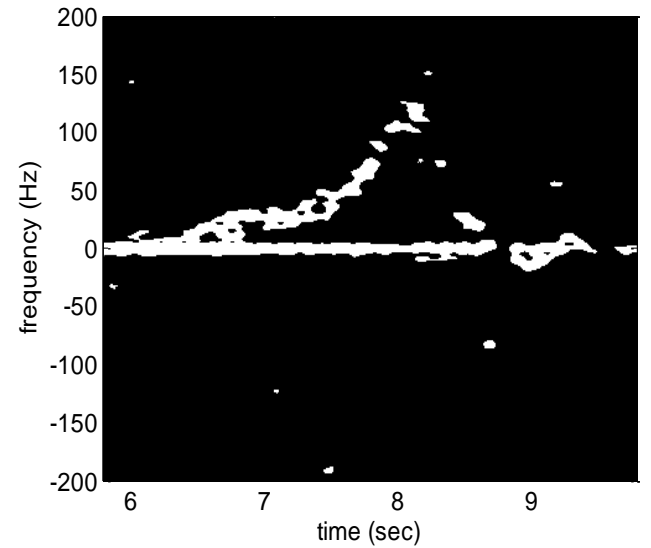

(a)

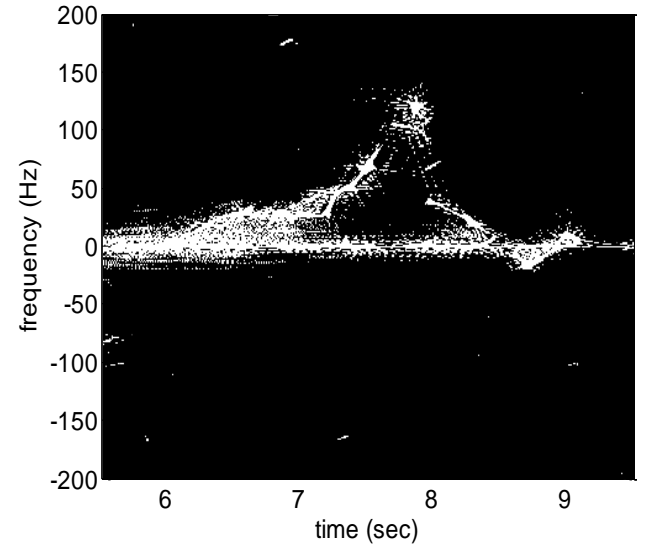

(b)

Figure 5. Segmented TFDs of falls with added artificial noise. (a) Spectrogram; (b) EMBD.

\section{CONCLUSION}

We have examined the offerings of using high-resolution time-frequency distributions, particularly the extended modified B-distribution, for the classification of fall detection. Analysis of real measurement data confirms that the EMBD provides improved contrast and connectivity in the resulting time-frequency distribution and reveals a higher level of details as compared with the coarse and smoothed time-frequency signatures offered by spectrograms.

\section{ACKNOWLEDGMENT}

This paper is made possible by NPRP Grant \# NPRP 6-680-2-282 from the Qatar National Research Fund (a member of Qatar Foundation). The statements made herein are solely the responsibility of the authors.

\section{REFERENCES}

[1] S. Tomii and T. Ohtsuki, "Falling detection using multiple Doppler sensors," in Proceedings of IEEE International Conference on e-Health Networking, Applications and Services, Beijing, China, pp. 196-201, Oct. 
2012.

[2] M. Chuah and F. Fu, "ECG anomaly detection via time series analysis," in Proceedings of International Symposium on Parallel and Distributed Processing and Applications, Niagara Falls, ON, Canada, Aug. 2007.

[3] A. Høst-Madsen, N. Petrochilos, O. Boric-Lubecke, V. M. Lubecke, B-K. Park, and Q. Zhou, "Signal processing methods for Doppler radar heart rate monitoring," in D. Mandic, M. Golz, A. Kuh, D. Obradovic, and T. Tanaka (Eds.), Signal Processing Techniques for Knowledge Extraction and Information Fusion, Springer, pp. 121-140, 2008.

[4] O. Aardal, S-E. Hamran, T. Berger, J. Hammerstad, and T. S. Lande, "Radar cross section of the human heartbeat and respiration in the 500MHz to $3 \mathrm{GHz}$ band," in Proceedings of IEEE Radio and Wireless Symposium, Phoenix, AZ, pp. 422-425, Jan. 2011.

[5] L. Liu, M. Popescu, M. Skubic, M. Rantz, T. Yardibi, and P. Cuddihy, "Automatic fall detection based on Doppler radar motion signature," in Proceedings of International Conference on Pervasive Computing Technologies for Healthcare, Dublin, Ireland, May 2011.

[6] M. Wu, X. Dai, Y. D. Zhang, B. Davidson, J. Zhang, and M. G. Amin, "Fall detection based on sequential modeling of radar signal time-frequency features," in Proceedings of IEEE International Conference on Healthcare Informatics, Philadelphia, PA, Sept. 2013.

[7] A. Gadde, M. G. Amin, Y. D. Zhang, and F. Ahmad, "Fall detection and classifications based on time-scale radar signal characteristics," in Proceedings of SPIE Radar Sensor Technology Conference, Baltimore, MD, May 2014.

[8] Q. Wu, Y. D. Zhang, W. Tao, and M. G. Amin, "Radar-based fall detection based on Doppler time-frequency signatures for assisted living," IET Radar, Sonar and Navigation, vol. 9, no. 2, pp. 164-172, Feb. 2015.

[9] B. Y. Su, K. C. Ho, M. J. Rantz, and M. Skubic, "Doppler radar fall activity detection using the wavelet transform," IEEE Transactions on Biomedical Engineering, vol. 62, no. 3, pp. 865-875, March 2015.

[10] M. G. Amin (Ed.), Through the Wall Radar Imaging. CRC Press, 2011.

[11] M. I. Skolnik, Introduction to Radar Systems, Third Edition. McGraw-Hill, 2001.

[12] V. Chen, The Micro-Doppler Effect in Radar. Artech House, 2011.

[13] T. Yardibi, P. Cuddihy, S. Genc, C. Bufi, M. Skubic, M. Rantz, L. Liu, and C. Phillips, "Gait characterization via pulse-Doppler radar," in Proceedings of Annual IEEE International Conference on Pervasive Computing and Communications: Workshop on Smart Environments to Enhance Health Care, Seattle, WA, pp. 662-667, March 2011.

[14] G. E. Smith, F. Ahmad, and M. G. Amin, "Micro-Doppler processing for ultra-wideband radar data," in Proceedings of SPIE Radar Sensor Technology XVI, Baltimore, MD, April 2012.

[15] M. Mercuri, D. Schreurs1, and P. Leroux, "SFCW microwave radar for in-door fall detection," in Proceedings of IEEE Topical Conference on Biomedical Wireless Technologies, Networks, and Sensing Systems, Santa Clara, CA, pp. 53-56, Jan. 2012.

[16] P. Setlur, F. Ahmad, and M. G. Amin, "Analysis of micro-Doppler signals using linear FM basis decomposition," in Proceedings of the SPIE Symposium on Defense and Security, Orlando, FL, April 2006.

[17] B. Boashash, Time-Frequency Signal Analysis and Processing: A comprehensive Reference. Elsevier, 2003.

[18] B. Boashash, N. A. Khana, and T. Ben-Jabeura, "Time-frequency features for pattern recognition using highresolution TFDs: A tutorial review," Digital Signal Processing, vol. 40, pp. 1-30, May 2015.

[19] M. G. Amin and W. Williams, "High spectral resolution time-frequency distribution kernels," IEEE Transactions on Signal Processing, vol. 46, pp. 2796-2804, Oct. 1998.

[20] B. Boashash, G. Azemi, and J. M. O'Toole, "Time-frequency processing of nonstationary signals: Advanced TFD design to aid diagnosis with highlights from medical applications," IEEE Signal Processing Magazine, vol. 30, pp. 108-119, Nov. 2013.

[21] M. G. Amin, "Time-frequency spectrum analysis and estimation for nonstationary random processes," in B. Boashash (ed.), Time-Frequency Signal Analysis: Methods and Applications, Longman Cheshire, 1992.

[22] G. Matz and F. Hlawatsch, "Nonstationary spectral analysis based on time-frequency operator symbols and underspread approximations," IEEE Transactions on Information Theory, vol. 52, no. 3, pp. 1067-1086, Mar. 2006. 